\title{
AVALIAÇÃO DE CARAMBOLA (Averrhoa carambola L.) DOS TIPOS DOCE E ÁCIDO PARA O PROCESSAMENTO DE FRUTA EM CALDA
}

\author{
PATRICIA PRATI * \\ JOÃO NUNES NOGUEIRA** \\ CARLOS TADEU DOS SANTOS DIAS***
}

\begin{abstract}
Frutas dos tipos doce e ácido de carambola foram analisadas fisicamente (textura e rendimento) e quimicamente $(\mathrm{pH}$, acidez total titulável, teor de ácido ascórbico e sólidos solúveis), a fim de determinar suas características para o processamento de fruta em calda. $\mathrm{Na}$ elaboração das frutas em calda diversos tratamentos químicos foram empregados (uso de xaropes com diferentes concentrações de sacarose, adição de pectina e $\mathrm{CaCl}_{2}$ em várias concentrações e de ácido ascórbico), visando avaliar a adequabilidade dos dois tipos de fruta de carambola ao processamento térmico. Os produtos foram submetidos à análises físicas (peso bruto, peso drenado, vácuo e textura), químicas $(\mathrm{pH}$, acidez total titulável, teor de ácido ascórbico e sólidos solúveis) e sensoriais (textura, sabor e cor). Quanto ao produto obtido com as frutas doces, a adição de $\mathrm{CaCl}_{2}$ e pectina foi efetiva na manutenção de sua textura, já para os produtos obtidos com as frutas ácidas esta prática não surtiu o mesmo efeito. Os tratamentos com adição de $\mathrm{CaCl}_{2}$ mostraram-se inferiores àquele cujo xarope foi adicionado somente de pectina, evidenciando que esse componente favorece a textura de frutas ácidas processadas. Os dois tipos de carambola estudados apresentaram, sob o ponto de vista tecnológico, adequadas características físicas e químicas. Entretanto, as frutas tipo doce mostraram-se mais apropriadas para a elaboração de fruta em calda, sendo superiores quanto à textura e sabor em relação às frutas ácidas.
\end{abstract}

PALAVRAS-CHAVE: CARAMBOLA-PROCESSAMENTO; FRUTA EM CALDA.

* M.Sc. em Ciência e Tecnologia de Alimentos, Doutoranda, Universidade Estadual de Campinas, Departamento de Tecnologia de Alimentos, Laboratório de Frutas e Produtos Açucarados, Campinas, SP (e-mail: pprati00@yahoo.com).

** Professor Doutor (Aposentado), Departamento de Agroindústria, Alimentos e Nutrição, Setor de Frutas e Hortaliças, Escola Superior de Agricultura "Luiz de Queiroz" (ESALQ), Universidade de São Paulo (USP), Piracicaba, SP.

*** Professor Doutor, Departamento de Matemática, ESALQ/USP, Piracicaba, SP. 


\section{INTRODUÇÃO}

A caramboleira (Averrhoa carambola L.) é uma planta tropical originária da Ásia. Seus frutos denominados carambola, ou em inglês "star fruit", são considerados exóticos devido ao formato de estrela da fruta quando cortada em secções transversais $(21,23)$.

A produção de carambolas constitui atividade altamente promissora sob o ponto de vista econômico, pela qualidade, sabor, formato único e atraente da fruta $(4 ; 17)$. A área de cultivo estende-se por todo o território nacional, porém o principal estado produtor é São Paulo (15).

A colheita manual de carambolas é muito complicada, pois as frutas sofrem danos facilmente, devido à epiderme fina, frágil e também ao seu formato nervado (4). Tratando-se de fruta extremamente delicada e perecível, a carambola deve ser cuidadosamente coletada, classificada, transportada e comercializada (15).

As frutas de caramboleira são climatéricas, e por esta razão podem ocorrer na pós-colheita mudanças na cor e nos teores de ácidos orgânicos e de sólidos solúveis (4). O principal ácido identificado na carambola foi o oxálico, cujo teor é maior na fruta madura e menor nas frutas do tipo doce (12).

Após a colheita, os alimentos estão sujeitos a alterações (biológica, química e física) que podem ocorrer no seu manuseio, transporte, processamento e armazenamento.

A principal enzima presente em frutas é a polifenoloxidase, que catalisa as reações oxidativas de compostos fenólicos a orto-quinonas, as quais polimerizam-se e/ou interagem com proteínas ou aminoácidos para produzir compostos escuros, como as melanoidinas. Essa seqüência de reações é conhecida como escurecimento enzímico e constitui um dos mais graves problemas do processamento de frutas e hortaliças (19). Ainda podem ser citadas as reações responsáveis pelo aparecimento de odores e sabores estranhos no produto final, que são catalisadas por enzimas oxidativas como a peroxidase e a catalase (24).

O emprego de calor (branqueamento) é o método mais usado para o controle do escurecimento enzímico, pois é mais efetivo na inativação da polifenoloxidase (6).

A aceitabilidade de determinado alimento no mercado depende muito de 
sua cor. Os alimentos apresentam cor característica que, quando desviada da aparência esperada sugere que o alimento está deteriorado e/ou processado inadequadamente (25).

A cor dos carotenos e carotenóides (pigmentos naturais) variam do amarelo ao vermelho escuro. São mais estáveis que as antocianinas, apresentam estabilidade na faixa de $\mathrm{pH}$ da maioria dos alimentos, não são destruídos por substâncias redutoras como o ácido ascórbico, sendo degradados por oxidação (11).

A textura dos produtos, atributo importante de qualidade, exerce influência nos hábitos alimentares e na preferência do consumidor, afetando o manuseio e o processamento dos alimentos (20). A textura de frutas e hortaliças é conferida por substâncias pécticas presentes nos seus tecidos, tais como ácidos pécticos, pectina e protopectina. Com o amadurecimento das frutas ou senescência das hortaliças, tais compostos perdem gradativamente sua textura tornando-se mais macios. Isso ocorre devido à degradação das substâncias pécticas por enzimas pécticas que podem ser endógenas (presentes em plantas superiores) ou produzidas por microrganismos (6). A adição ou imersão de produtos de frutas em sais de cálcio proporciona maior firmeza aos tecidos devido à formação de pectato de cálcio resultante da interação do cálcio com a pectina degradada na lamela média (14).

Como a produção dos alimentos é sazonal buscam-se meios de conservação para que se possa consumí-los por longos períodos com propriedades semelhantes ao que apresentavam quando "in natura" (8). A conservação de alimentos pode ser definida como todo método de tratamento que seja capaz de prolongar sua duração, mantendo grau aceitável de qualidade, incluindo cor, textura e aroma (10).

Sob o ponto de vista econômico, a conservação de frutas pelo calor é o processo que assume maior importância (9). O objetivo principal do tratamento térmico é assegurar a destruição somente dos organismos vivos potencialmente deterioradores dos alimentos ou capazes de prejudicar a saúde do consumidor, de tal forma que este tratamento seja eficiente microbiologicamente sem afetar os caracteres organolépticos do produto $(3,7)$. Dentre os métodos de conservação de alimentos pelo calor a apertização constitui o de maior importância industrial, mundialmente empregado não só na grande como também na média e pequena indústria.

Dentre os diversos produtos que podem ser obtidos das frutas destaca- 
se a fruta em calda, preferida pelas indústrias de conservas e de larga aceitação pelos consumidores em todos os continentes (30).

$\mathrm{Na}$ Guiana, a carambola é processada como fruta fervida em xarope de açúcar (compota). Pesquisas têm indicado que produto aceitável pode ser preparado por desidratação osmótica em xarope de açúcar de concentração reforçada (13). De acordo com estudos realizados a carambola apresenta potencial para utilização em combinação com sucos de frutas tropicais (18).

Esta pesquisa teve como objetivo estudar alternativas de consumo de carambolas doces e ácidas na forma de fruta em calda, visando aproveitamento do excedente de produção não aceito pelo mercado consumidor. Desta forma, além de reduzir o prejuízo na produção, seria possível estimular a expansão da cultura no país criando-se alternativas de processamento e comercialização dos frutos.

\section{MATERIAL E MÉTODOS}

As atividades de pesquisa foram desenvolvidas no Setor de Processamento de Frutas e Hortaliças do Departamento de Agroindústria, Alimentos e Nutrição da Escola Superior de Agricultura "Luiz de Queiroz" (USP). Como matéria-prima foram utilizadas carambolas do tipo doce e ácido, plenamente desenvolvidas, com coloração predominantemente amarelada, provenientes da região de Mirandópolis, Estado de São Paulo.

\subsection{CARACTERIZAÇÃO DA MATÉRIA-PRIMA}

A fim de caracterizar os dois tipos de matéria-prima foram realizadas avaliações físicas e químicas da mesma, utilizando-se para cada análise duas amostras.

\subsubsection{Análises Físicas}

Determinou-se o rendimento da matéria-prima com amostras de $1000 \mathrm{~g}$ de frutas, tomadas aleatoriamente dos lotes do material. As carambolas de cada amostra foram pesadas em balança semi-analítica, lavadas, drenadas, fatiadas, sendo as sementes retiradas e as plantas pesadas novamente. Os resultados foram apresentados em porcentagem do peso 
da matéria-prima pronta para o processamento, em relação ao peso original da matéria-prima.

Determinou-se a textura da matéria-prima em "Texture Testing System" modelo TP-2, acoplado a registrador automático de variações de força, operando com célula-padrão de cisalhamento e compressão CS-1, com 10 lâminas de $1 / 8$ polegada de espessura e ângulos de $90^{\circ}$. Usou-se anel de prova de $300 \mathrm{lbf}$ e velocidade de descida do pistão de $20 \mathrm{~cm} / \mathrm{min}$. As amostras foram anteriormente pesadas (50 gramas de fruta) e colocadas ao acaso na célula-teste. Os resultados foram expressos em lbf/g de amostra.

\subsubsection{Análises Químicas}

Determinou-se o pH da matéria-prima em potenciômetro Alphalab, modelo PA-200, sendo os resultados expressos em unidades de $\mathrm{pH}$.

A acidez total titulável da matéria-prima foi determinada segundo métodologia descrita pela AOAC (1).

Determinou-se o teor de sólidos solúveis em refratômetro Atago, modelo $\mathrm{N}-1$, sendo os resultados expressos em ${ }^{\circ}$ Brix.

O teor de ácido ascórbico foi determinado por extração em ácido oxálico e titulação, segundo método de Tillmans modificado por LEME JÚNIOR e MALAVOLTA (16).

\subsection{PROCESSAMENTO}

A seqüência de operações utilizada no processamento de carambola em calda está indicada na Figura 1.

\subsubsection{Tratamentos de Pré-Processamento}

As frutas de cada tipo de carambola foram submetidas aos tratamentos que constam na Figura 2. 


\section{FIGURA 1 - FLUXOGRAMA DAS OPERAÇÕES UTILIZADAS NO PROCESSAMENTO DAS CARAMBOLAS}

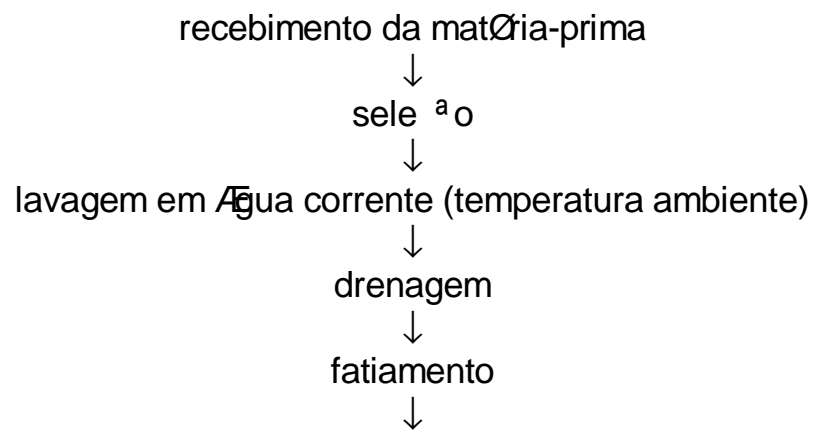

remo a o manual das sementes $\rightarrow$ retirada de amostras para an/Fise $\downarrow$ tratamentos de prøprocessamento $\downarrow$ acondicionamento

$\downarrow$ exaust ${ }^{\mathrm{a}}$ o $(80$ "C)<smiles>[AlH2]</smiles>
recrava $\stackrel{a}{0}$ esteriliza ำ $\stackrel{\downarrow}{\downarrow}(97$ " C/15 minutos)

$\downarrow$

resfriamento (tanque com circula $\stackrel{\text { a }}{\sim}$ o de Agua a temperatura ambiente) $\downarrow$ armazenamento a temperatura ambiente

\subsubsection{Acondicionamento}

$\mathrm{O}$ acondicionamento foi efetuado em latas de folha-de-flandres, com capacidade para $800 \mathrm{~g}$ de produto $(99,5 \times 118 \mathrm{~mm})$, revestidas internamente com verniz epóxi, próprias para produtos ácidos. Em cada lata foram colocados $330 \mathrm{~g}$ de carambola, preenchendo-se o volume do recipiente com o xarope (espaço livre de $5 \mathrm{~mm}$ ). 


\subsubsection{Exaustão e Recravação}

Efetuou-se a exaustão em banho-maria, até que o centro geométrico dos recipientes atingisse $80^{\circ} \mathrm{C}$. A seguir as latas foram imediatamente recravadas.

\subsubsection{Esterilização, Resfriamento e Armazenamento}

A esterilização ocorreu por imersão das latas em água fervente $\left(97^{\circ} \mathrm{C}\right)$, durante 15 minutos, seguida de resfriamento em tanque com circulação de água a temperatura ambiente. As latas foram armazenadas em temperatura ambiente.

\section{FIGURA 2- ESQUEMA DOS TRATAMENTOS DE PRÉ- PROCESSAMENTO AOS QUAIS AS FRUTAS DE CADA TIPO DE CARAMBOLA FORAM SUBMETIDAS}

\begin{tabular}{|c|c|c|c|c|c|}
\hline \multicolumn{3}{|c|}{ Sem adi a o de C AEcio } & \multicolumn{3}{|c|}{ Com adi a o de C Atcio } \\
\hline 1 & 2 & 3 & 4 & 5 & 6 \\
\hline Carambola & Carambola & Carambola & Carambola & Carambola & Carambola \\
\hline+ & + & + & + & + & + \\
\hline Xarope de & Xarope de & Xarope de & Xarope de & Xarope de & Xarope de \\
\hline Sacarose & Sacarose & Sacarose & Sacarose & Sacarose & Sacarose \\
\hline (Brix de & (Brix de & (Brix de & (Brix de & (Brix de & (Brix de \\
\hline equil brio & equil brio & equil brio & equil brio & equil brio & equil brio \\
\hline \multirow[t]{12}{*}{$22 ")$} & $25 ")$ & $28 ”)$ & $22 ")$ & $25 ")$ & $28 ")$ \\
\hline & + & + & + & + & + \\
\hline & Pectina & Pectina & $\mathrm{CaCl}_{2}$ & Pectina & Pectina \\
\hline & $(0,1 \%)$ & $(0,2 \%)$ & $(0,1 \%)$ & $(0,1 \%)$ & $(0,2 \%)$ \\
\hline & & + & & + & + \\
\hline & & 'cido & & $\mathrm{CaCl}_{2}$ & $\mathrm{CaCl}_{2}$ \\
\hline & & asc rbico & & $(0,2 \%)$ & $(0,4 \%)$ \\
\hline & & $(300 \mathrm{mg} / \mathrm{kg})$ & & & \\
\hline & & & & & + \\
\hline & & & & & cido \\
\hline & & & & & asc rbico \\
\hline & & & & & $(300 \mathrm{mg} / \mathrm{kg})$ \\
\hline
\end{tabular}




\subsection{AVALIAÇÃO DO PRODUTO PROCESSADO}

Análises físicas e químicas do produto foram efetuadas aos 30, 60, 90, 120,150 e 180 dias após o processamento, enquanto que as avaliações sensoriais ocorreram aos $0,30,60,90,120,150$ e 180 dias após 0 processamento.

\subsubsection{Análises físicas}

A textura do produto foi determinada conforme descrito no item 2.1.1 e o vácuo com o auxílio do Vacuômetro "Marshalltown", em polegadas de mercúrio (pol.Hg).

Determinou-se o peso bruto em balança semi-analítica (peso da embalagem mais o peso do produto).

O peso drenado do produto foi determinado em conjunto de peneira USTyler $n^{\circ} 8$ (abertura de malha de 2,38 mm) e fundo. Todo conteúdo da lata foi vertido no conjunto peneira-fundo o deixado para drenar por 2 minutos, inclinando-se ligeiramente a peneira (ângulo de 45ㅇ) sobre o fundo. $O$ peso da peneira mais o peso do produto menos o da peneira constituiu o peso drenado.

\subsubsection{Análises químicas}

Determinou-se o pH, a acidez total titulável, os teores de sólidos solúveis e de ácido ascórbico conforme descrito no item 2.1.2, utilizando o xarope do produto.

\subsubsection{Análise sensorial}

A análise sensorial englobou avaliações subjetivas de cor, textura e sabor, realizadas por equipe de 8 julgadores, previamente selecionados e instruídos para esse tipo de avaliação, considerando que se trata de produto ainda não existente no mercado. Utilizou-se escala numérica estruturada de nove pontos (17), cujos parâmetros dos atributos foram definidos e fornecidos aos provadores (Tabela 1). 


\section{TABELA 1 - DEFINIÇÃO DOS PARÂMETROS DOS ATRIBUTOS DA ANÁLISE SENSORIAL}

\begin{tabular}{|c|c|c|}
\hline Textura & $\begin{array}{l}\text { Muito mole } \\
\text { Mole } \\
\text { Nem mole nem firme } \\
\text { Firme } \\
\text { Muito firme }\end{array}$ & $\begin{array}{l}\text { Quando a fruta estAEdesintegrada } \\
\text { Com textura branda mas } n^{a} \text { o desintegrada } \\
\text { Com textura intermediffia (entre mole } \\
\text { firme) } \\
\text { Com textura firme mas } n^{3} 0 \text { crocante } \\
\text { Com textura crocante }\end{array}$ \\
\hline Sabor & $\begin{array}{l}\text { Sem sabor } \\
\text { Sabor fraco } \\
\text { Sabor moderado } \\
\text { Sabor intenso } \\
\text { Sabor muito intenso }\end{array}$ & $\begin{array}{l}\text { Isenta de saborda fruta } \\
\text { Com sabor levemente da fruta } \\
\text { Com saborda fruta } \\
\text { Com bastante sabor da fruta } \\
\text { Com intenso saborda fruta }\end{array}$ \\
\hline Cor & $\begin{array}{l}\text { Sem cor } \\
\text { Cor fraca } \\
\text { Cormoderada } \\
\text { Cor intensa } \\
\text { Cormuito intensa }\end{array}$ & $\begin{array}{l}\text { Com cor esbranqui ada } \\
\text { Com corlevemente am arela } \\
\text { Com cor amarela } \\
\text { Com corintensamente amarela } \\
\text { Com cortendendo ao alaranjado }\end{array}$ \\
\hline
\end{tabular}

Para avaliação da cor das frutas, as amostras foram servidas em pratos plásticos brancos, dispostos aleatoriamente em bandeja de alumínio, em local com iluminação de luz fluorescente. Os pratos foram codificados com números aleatórios compostos de três dígitos. Para avaliação da textura e do sabor das frutas adotou-se o mesmo procedimento anterior porém utilizando-se iluminação vermelha para mascarar a cor da fruta.

Todas as avaliações foram efetuadas com duas repetições, de forma que cada provador avaliasse a mesma amostra duas vezes (em sessões diferentes).

\subsection{ANÁLISE ESTATÍSTICA DOS RESULTADOS}

Os dados da matéria-prima foram apresentados através de médias, com os respectivos erros padrões da média, correspondente a duas repetições. Já, os dados do produto processado foram analisados mediante delineamento em blocos casualizados (DBC), com seis tratamentos e duas repetições para cada tipo de fruta testada, utilizando-se teste de Tukey ao nível de 5\% de significância (28). 
As médias obtidas na avaliação sensorial foram analisadas pelo DBC, com seis tratamentos e 16 repetições para cada tipo de fruta e teste de Tukey ao nível de $5 \%$ de significância (28).

\section{RESULTADOS E DISCUSSÃO}

\subsection{CARACTERIZAÇÃO DA MATÉRIA-PRIMA}

Os resultados das avaliações dos atributos físicos da matéria-prima são apresentados na Tabela 2.

TABELA 2 - CARACTERÍSTICAS FÍSICAS DA MATÉRIA-PRIMA

\begin{tabular}{l|c|c}
\hline \multicolumn{1}{c|}{ AnAFses } & Tipo Doce & Tipo` cido \\
\hline Textura (lbf/g) & $3,44 \pm 0,220$ & $3,31 \pm 0,290$ \\
Rendimento (\%) & $84,20 \pm 0,640$ & $85,46 \pm 0,245$ \\
\hline
\end{tabular}

Verificou-se que as frutas do tipo doce apresentaram textura mais firme (3,44 lbf/g) que as do tipo ácido (3,31 lbf/g), no entanto, com relação ao rendimento exibiram resultado inferior $(84,20 \%)$. O rendimento dos dois tipos de fruta de carambola não diferiram muito entre si, pois para cada $1000 \mathrm{~g}$ de carambola "in natura", $842 \mathrm{~g}$ do tipo doce e 854,6 g do tipo ácido foram utilizadas para o processamento. Tais resultados representam qualidade e adequado aproveitamento da matéria-prima, ou seja, pequena presença de frutas amassadas e/ou deterioradas por microrganismos ou insetos.

Os resultados das determinações de teor de ácido ascórbico, $\mathrm{pH}$, acidez total titulável e teor de sólidos solúveis (Brix) da matéria-prima estão apresentados na Tabela 3.

Os teores de vitamina $\mathrm{C}$, relativos aos dois tipos de fruta estudados, mostraram-se bastante superiores ao obtido por OLIVEIRA et al. (23) em carambolas, cujo tipo não foi especificado (17,16 mg/100 g). Comparando os dados da Tabela 3 verifica-se que as frutas do tipo doce são mais ricas 
em vitamina $C$ que as do tipo ácido.

TABELA 3 - CARACTERÍSTICAS QUÍMICAS DA MATÉRIA-PRIMA

\begin{tabular}{l|c|c}
\hline \multicolumn{1}{c|}{ An/Fises } & Tipo Doce & Tipo `cido \\
\hline Vitamina C (mg/100 g) & $44,28 \pm 1,425$ & $42,86 \pm 0$ \\
pH & $4,01 \pm 0,095$ & $2,52 \pm 0,055$ \\
Acidez total titul/Fel (\% Aeido ox/\#ico) & $0,10 \pm 0,002$ & $0,31 \pm 0,005$ \\
Teor de s lidos soloreis ("Brix) & $8,25 \pm 0,250$ & $7,75 \pm 0,250$ \\
\hline
\end{tabular}

Os valores de $\mathrm{pH}$ obtidos foram muito semelhantes aos encontrados por JOSEPH e MENDONCA (13), 2,40 para fruta do tipo ácido e 4,00 para o tipo doce. Já OLIVEIRA et al. (23) encontraram valor intermediário $(3,33)$, porém não especificaram o tipo de fruta analisada.

Valores elevados de sólidos solúveis foram encontrados para as frutas doce e ácida. O resultado obtido para o tipo doce mostrou-se semelhante ao citado por JOSEPH e MENDONCA (12) que encontraram valores entre 6,0 e 8,4 ํㅏix. No entanto, em relação ao tipo ácido o resultado obtido foi superior aos desses autores (valores entre 3,4 e 5,0 ${ }^{\circ}$ Brix) e de OLIVEIRA et al. (23), que encontraram 5,1 ํㅜㅅㅜ mas não especificaram o tipo de fruta estudado.

As frutas doces e ácidas apresentaram valores de acidez total titulável ( $\mathrm{g}$ de ácido oxálico/100 g de amostra) diferenciados. Para o tipo doce o resultado mostrou-se semelhante ao citado por JOSEPH e MENDONCA (12), já em relação ao tipo ácido foi inferior (valores entre 0,57 e 0,63). OLIVEIRA et al. (23) obtiveram 0,37 g de ácido cítrico/100 g de amostra, porém não especificaram o tipo de fruta estudado.

\subsection{CARACTERIZAÇÃO DO PRODUTO FINAL}

\subsubsection{Análises Físicas}

A análise física mais relevante para o trabalho foi a determinação da textura (Tabelas 4a e 4b), as demais visaram acompanhar o armazenamento do produto conforme é realizado em grandes empresas alimentícias. 
TABELA 4a - TEXTURA (Ibf/g de amostra) DAS CARAMBOLAS DOCES EM CALDA

\begin{tabular}{|c|c|c|c|c|c|c|c|}
\hline poca & T1 & T2 & T3 & T4 & T5 & T6 & Mødias \\
\hline 30 & 1,86 & 2,07 & 1,98 & 2,24 & 1,72 & 1,90 & $1,96^{b}$ \\
\hline 60 & 1,78 & 2,11 & 2,32 & 2,45 & 3,34 & 3,04 & $2,51^{\mathrm{a}}$ \\
\hline 90 & 1,99 & 2,14 & 2,32 & 2,14 & 2,71 & 2,87 & $2,36^{\mathrm{a}}$ \\
\hline 120 & 1,83 & 2,09 & 2,27 & 2,32 & 2,83 & 3,37 & $2,45^{a}$ \\
\hline 150 & 2,04 & 2,25 & 2,26 & 2,57 & 2,26 & 3,33 & $2,45^{\mathrm{a}}$ \\
\hline 180 & 2,08 & 1,86 & 2,18 & 2,11 & 2,24 & 3,39 & $2,31^{\mathrm{ab}}$ \\
\hline Mødias & $1,93^{\mathrm{D}}$ & $2,09^{C D}$ & $2,22^{\mathrm{BC}}$ & $2,30^{B C}$ & $2,52^{\mathrm{B}}$ & $2,98^{\mathrm{A}}$ & \\
\hline F(Trat.) & \multicolumn{7}{|c|}{$17,52^{*}$} \\
\hline$F($ poca $)$ & \multicolumn{7}{|c|}{$35,29^{*}$} \\
\hline
\end{tabular}

* significativo ao nível de $1 \%$.

Médias seguidas pela mesma letra não diferem entre si ao nível de 5\%.

Com relação à textura, as frutas do tipo doce apresentaram resultados maiores que as do tipo ácido. Observou-se que para as frutas doces, o tratamento 6 (maior valor encontrado) diferiu significativamente dos demais, já que o xarope foi adicionado de $0,2 \%$ de pectina e $0,4 \%$ de $\mathrm{CaCl}_{2} \mathrm{em}$ relação ao peso da fruta. Ainda para esse tipo de fruta, o menor valor de textura foi encontrado para o tratamento 1 (testemunha), que só não diferiu do tratamento 2 .

Em relação às frutas ácidas o maior valor de textura foi obtido pelo tratamento 2 (adição ao xarope de $0,1 \%$ de pectina em relação ao peso da fruta), que diferiu estatisticamente dos demais, sendo os menores valores encontrados para os tratamentos 4 e 5 (adição de $0,1 \%$ de $\mathrm{CaCl}_{2}$, e $0,1 \%$ de pectina $+0,2 \%$ de $\mathrm{CaCl}_{2}$, respectivamente). Tais resultados evidenciaram que o cálcio não foi efetivo para preservar a textura das frutas ácidas processadas, conforme observado por RICHARDS (26) em morango em calda. Esses resultados podem ser explicados pela alta acidez da fruta. A acidificação da parede celular e da lamela média pode promover a substituição de $\mathrm{Ca}^{2+}$ pelo $\mathrm{H}^{+}$e, conseqüentemente, 0 enfraquecimento das ligações e da coesão existentes entre as cadeias de pectina da parede celular e da lamela média (2). 
TABELA 4b - TEXTURA (Ibf/g de amostra) DAS CARAMBOLAS ÁCIDAS EM CALDA

\begin{tabular}{c|c|c|c|c|c|c|c}
\hline poca & T1 & T2 & T3 & T4 & T5 & T6 & MØdias \\
\hline $\mathbf{3 0}$ & 1,14 & 1,45 & 1,11 & 0,91 & 1,03 & 1,04 & $1,11^{\mathrm{C}}$ \\
$\mathbf{6 0}$ & 1,36 & 1,31 & 1,20 & 1,01 & 1,01 & 1,15 & $1,17^{\mathrm{bc}}$ \\
$\mathbf{9 0}$ & 1,35 & 1,44 & 1,23 & 1,15 & 1,35 & 1,30 & $1,30^{\mathrm{ab}}$ \\
$\mathbf{1 2 0}$ & 1,24 & 1,36 & 1,26 & 1,15 & 1,13 & 1,40 & $1,26^{\mathrm{abc}}$ \\
$\mathbf{1 5 0}$ & 1,26 & 1,56 & 1,40 & 1,14 & 1,32 & 1,39 & $1,34^{\mathrm{a}}$ \\
$\mathbf{1 8 0}$ & 1,26 & 1,53 & 1,25 & 1,25 & 1,18 & 1,25 & $1,29^{\mathrm{ab}}$ \\
MØdias & $1,27^{\mathrm{B}}$ & $1,44^{\mathrm{A}}$ & $1,24^{\mathrm{B}}$ & $1,10^{\mathrm{C}}$ & $1,17^{\mathrm{BC}}$ & $1,25^{\mathrm{B}}$ & \\
\hline $\mathrm{F}$ (Trat.) & \multicolumn{7}{|c}{$12,10^{*}$} \\
$\mathrm{~F}$ ( poca) & $5,20^{*}$ \\
\hline
\end{tabular}

* significativo ao nível de $1 \%$.

Médias seguidas pela mesma letra não diferem entre si ao nível de $5 \%$.

De maneira geral, para os dois tipos de fruta houve incremento da textura até os 90 dias de armazenamento, mantendo-se praticamente inalterada até 180 dias de estocagem.

Comparando-se os valores das Tabelas 2, 4a e 4b para os dois tipos de fruta pode-se observar que a temperatura exerce efeito drástico sobre a textura. O mesmo foi observado por RICHARDS (26) trabalhando com morango em calda.

\subsubsection{Análises químicas}

Os valores do teor de sólidos solúveis não variaram já que os xaropes foram preparados de modo a obter determinadas concentrações de equilíbrio na conserva (22 ${ }^{\circ}$ Brix para os tratamentos 1 e 4,25 B Brix para os tratamentos 2 e 5 , e 28 Brix para os tratamentos 3 e 6 ).

Quanto ao parâmetro pH (Tabelas $5 \mathrm{a}$ e $5 \mathrm{~b}$ ) para as frutas doces, os valores não diferiram entre si aos 30 e 60 dias mas sim em relação às outras épocas de avaliação. Para o tipo ácido não houve diferença significativa entre os tempos de armazenamento. 
TABELA 5a - pH DAS CARAMBOLAS DOCES EM CALDA

\begin{tabular}{c|c|c|c|c|c|c|c}
\hline poca & T1 & T2 & T3 & T4 & T5 & T6 & MØdias \\
\hline $\mathbf{3 0}$ & 3,72 & 3,67 & 3,57 & 3,36 & 3,34 & 3,07 & $3,45^{\mathrm{b}}$ \\
$\mathbf{6 0}$ & 3,65 & 3,60 & 3,53 & 3,32 & 3,28 & 3,00 & $3,40^{\mathrm{b}}$ \\
$\mathbf{9 0}$ & 3,81 & 3,64 & 3,72 & 3,46 & 3,38 & 3,21 & $3,54^{\mathrm{a}}$ \\
$\mathbf{1 2 0}$ & 3,86 & 3,77 & 3,76 & 3,48 & 3,41 & 3,21 & $3,58^{\mathrm{a}}$ \\
$\mathbf{1 5 0}$ & 3,83 & 3,73 & 3,73 & 3,44 & 3,40 & 3,19 & $3,55^{\mathrm{a}}$ \\
$\mathbf{1 8 0}$ & 3,88 & 3,79 & 3,71 & 3,47 & 3,38 & 3,20 & $3,57^{\mathrm{a}}$ \\
MØdias & $3,79^{\mathrm{A}}$ & $3,70^{\mathrm{AB}}$ & $3,67^{\mathrm{B}}$ & $3,42^{\mathrm{C}}$ & $3,36^{\mathrm{C}}$ & $3,15^{\mathrm{D}}$ & \\
\hline F(Trat.) & \multicolumn{7}{|c}{$192,74^{\star}$} \\
F( poca) & $18,75^{\star}$ \\
\hline
\end{tabular}

* significativo ao nível de $1 \%$.

Médias seguidas pela mesma letra não diferem entre si ao nível de 5\%.

\section{TABELA 5b - pH DAS CARAMBOLAS ÁCIDAS EM CALDA}

\begin{tabular}{c|c|c|c|c|c|c|c}
\hline poca & T1 & T2 & T3 & T4 & T5 & T6 & M Ødias \\
\hline $\mathbf{3 0}$ & 2,31 & 2,24 & 2,22 & 2,11 & 1,94 & 2,11 & $2,15^{\mathrm{b}}$ \\
$\mathbf{6 0}$ & 2,31 & 2,23 & 2,33 & 2,05 & 1,86 & 1,82 & $2,10^{\mathrm{b}}$ \\
$\mathbf{9 0}$ & 2,28 & 2,27 & 2,26 & 2,00 & 1,90 & 1,62 & $2,05^{\mathrm{b}}$ \\
$\mathbf{1 2 0}$ & 2,42 & 2,25 & 2,32 & 2,07 & 1,87 & 1,70 & $2,10^{\mathrm{b}}$ \\
$\mathbf{1 5 0}$ & 2,33 & 2,30 & 2,39 & 2,03 & 1,90 & 1,87 & $2,14^{\mathrm{b}}$ \\
$\mathbf{1 8 0}$ & 2,31 & 2,21 & 2,34 & 2,10 & 1,93 & 1,81 & $2,12^{\mathrm{b}}$ \\
Mødias & $2,33^{\mathrm{A}}$ & $2,25^{\mathrm{A}}$ & $2,31^{\mathrm{A}}$ & $2,06^{\mathrm{B}}$ & $1,90^{\mathrm{C}}$ & $1,82^{\mathrm{C}}$ & \\
\hline F(Trat.) & \multicolumn{7}{|c}{$35,76^{*}$} \\
F( poca) & \multicolumn{7}{|c}{$36,50^{*}$} \\
\hline
\end{tabular}

* significativo ao nível de $1 \%$.

Médias seguidas pela mesma letra não diferem entre si ao nível de 5\%.

Verificou-se que o tratamento 6 para fruta tipo doce foi o de menor valor, diferindo dos demais. O tratamento 1 exibiu o maior valor, não diferindo do tratamento 2, mas dos demais. Já, para o tipo ácido, os tratamentos 1, 2 e 3 apresentaram os maiores valores, diferindo dos outros, porém não entre si. Os menores resultados foram obtidos pelos tratamentos 5 e 6 , que também não diferiram entre si, mas dos demais. $O$ tratamento 4 
apresentou valor intermediário e diferiu significativamente dos outros.

Observou-se que os valores de $\mathrm{pH}$ da fruta "in natura", tanto doce como ácida, foram superiores aos das processadas. Já RICHARDS (26) encontrou valores semelhantes entre matéria-prima e produto processado (morango em calda) durante todo o período de armazenamento.

As Tabelas 6a e 6b mostram os teores de ácido ascórbico dos produtos processados. Observou-se que para a fruta do tipo doce os tratamentos 3 e 6 foram superiores aos demais, diferindo desses, mas não entre si, o que pode ser justificado pela adição de vitamina $\mathrm{C}$ ao xarope dos referidos tratamentos. O menor valor foi observado para o tratamento 1 (testemunha). Os tratamentos 3 e 6 também foram superiores aos demais, no caso do tipo ácido, pelo mesmo motivo anteriormente citado, sendo que os mesmos diferiram entre si e dos demais. TABELA 6a - TEOR DE VITAMINA C (mg de ácido ascórbico/100 g
de amostra) DAS CARAMBOLAS DOCES EM CALDA

\begin{tabular}{|c|c|c|c|c|c|c|c|}
\hline poca & T1 & T2 & T3 & T4 & T5 & T6 & Mødias \\
\hline 30 & 42,05 & 42,46 & 71,78 & 42,78 & 42,85 & 70,51 & $52,07^{a}$ \\
\hline 60 & 34,36 & 38,36 & 60,26 & 38,62 & 39,47 & 65,13 & $46,03^{b}$ \\
\hline 90 & 34,21 & 36,84 & 54,87 & 34,36 & 38,72 & 61,54 & $43,42^{b}$ \\
\hline 120 & 26,31 & 31,58 & 49,74 & 34,21 & 38,46 & 49,74 & $38,34^{c}$ \\
\hline 150 & 25,64 & 29,28 & 47,37 & 30,00 & 35,26 & 48,42 & $35,99^{c}$ \\
\hline 180 & 24,52 & 27,41 & 40,53 & 28,36 & 32,29 & 44,37 & $32,91^{\mathrm{c}}$ \\
\hline Mødias & $31,18^{C}$ & $34,32^{\mathrm{B}}$ & $54,09^{A}$ & $34,72^{\mathrm{B}}$ & $37,84^{\mathrm{B}}$ & $56,61^{A}$ & \\
\hline F(Trat.) & \multicolumn{7}{|c|}{$54,10^{*}$} \\
\hline$F($ poca $)$ & \multicolumn{7}{|c|}{$88,78^{*}$} \\
\hline
\end{tabular}

* significativo ao nível de $1 \%$.

Médias seguidas pela mesma letra não diferem entre si ao nível de 5\%.

Com relação aos teores de vitamina $\mathrm{C}$, ao longo do período de armazenamento, verificou-se decréscimo de $36,80 \%$ para fruta do tipo doce e $29,88 \%$ para o tipo ácido. Tal fato também foi observado por NOVAES (22) na elaboração de néctares comum e dietético de morango, por SIVIERI (29) em geléias comum e dietética de morango, e por RICHARDS (26). A redução no teor de ácido ascórbico pode ser explicada 
pela oxidação desse componente durante o armazenamento. O calor acelera a oxidação especialmente quando o alimento está em contato com cobre, ferro ou enzimas oxidativas.

TABELA 6b - TEOR DE VITAMINA C (mg de ácido ascórbico/100 g de amostra) DAS CARAMBOLAS ÁCIDAS EM CALDA

\begin{tabular}{|c|c|c|c|c|c|c|c|}
\hline poca & T1 & T2 & T3 & T4 & T5 & T6 & Mødias \\
\hline 30 & 39,74 & 41,03 & 70,41 & 41,03 & 40,53 & 79,48 & $52,04^{\mathrm{ab}}$ \\
\hline 60 & 37,89 & 39,47 & 65,13 & 37,18 & 38,72 & 77,40 & $49,30^{b}$ \\
\hline 90 & 35,90 & 36,30 & 60,49 & 36,84 & 37,90 & 76,31 & $47,29^{b}$ \\
\hline 120 & 32,92 & 34,76 & 54,85 & 35,90 & 35,70 & 66,67 & $43,47^{\circ}$ \\
\hline 150 & 28,53 & 29,42 & 50,76 & 34,21 & 34,71 & 65,22 & $40,47^{c d}$ \\
\hline 180 & 24,42 & 26,20 & 46,15 & 32,35 & 32,38 & 57,43 & $36,49^{d}$ \\
\hline M Ødia s & $33,23^{\mathrm{D}}$ & $34,53^{C D}$ & $57,96^{\mathrm{B}}$ & $36,25^{\mathrm{C}}$ & $36,67^{\mathrm{C}}$ & $70,42^{\mathrm{A}}$ & \\
\hline$F$ (Trat.) & \multicolumn{7}{|c|}{$102,71^{*}$} \\
\hline$F($ poca $)$ & \multicolumn{7}{|c|}{$77,68^{*}$} \\
\hline
\end{tabular}

* significativo ao nível de $1 \%$.

Médias seguidas pela mesma letra não diferem entre si ao nível de 5\%.

SALOMÓN et al. (27) afirmam que perdas de ácido ascórbico são comuns em produtos armazenados, em temperatura ambiente, por períodos prolongados. ELEUTÉRIO (5) observou em geléia de acerola perda de $15,66 \%$ de ácido ascórbico, após o processamento e de 13,76\% após 6 meses de armazenamento. Mesmo assim, os valores obtidos ao final dos 180 dias de estocagem foram relativamente altos $(32,91 \mathrm{mg} / 100 \mathrm{~g}$ para o tipo doce e $36,49 \mathrm{mg} / 100 \mathrm{~g}$ para o tipo ácido).

A acidez total titulável (Tabelas 7a e 7b), para os dois tipos de fruta, a partir dos 30 dias de armazenamento evidenciou decréscimo nos valores de acidez, o que pode ser explicado pela redução no teor de ácido ascórbico, já que o mesmo é um dos componentes da acidez total titulável. Esse fato foi também observado por NOVAES (22), RICHARDS (26) e por SIVIERI (29).

Quanto aos tratamentos, para a fruta do tipo doce, o tratamento 6 evidenciou maior valor, sendo o menor resultado obtido para o tratamento 1 (que diferiu significativamente dos demais). No caso das frutas ácidas, 0 tratamento 6 também mostrou-se superior não diferindo apenas do 5 . Os menores valores foram encontrados para os tratamentos 1 e 2 , que não diferiram entre si nem do tratamento 3. 
TABELA 7a - ACIDEZ TOTAL TITULÁVEL ( $\mathrm{g}$ de ácido oxálico/100 g de amostra) DAS CARAMBOLAS DOCES EM CALDA

\begin{tabular}{c|c|c|c|c|c|c|c}
\hline poca & T1 & T2 & T3 & T4 & T5 & T6 & MØdias \\
\hline $\mathbf{3 0}$ & 0,094 & 0,105 & 0,117 & 0,108 & 0,121 & 0,130 & $0,112^{\mathrm{b}}$ \\
$\mathbf{6 0}$ & 0,085 & 0,101 & 0,112 & 0,110 & 0,108 & 0,123 & $0,106^{\mathrm{bc}}$ \\
$\mathbf{9 0}$ & 0,081 & 0,096 & 0,105 & 0,103 & 0,105 & 0,105 & $0,099^{\mathrm{cd}}$ \\
$\mathbf{1 2 0}$ & 0,083 & 0,085 & 0,100 & 0,094 & 0,105 & 0,114 & $0,097^{\mathrm{d}}$ \\
$\mathbf{1 5 0}$ & 0,092 & 0,101 & 0,108 & 0,090 & 0,101 & 0,130 & $0,090^{\mathrm{bcd}}$ \\
$\mathbf{1 8 0}$ & 0,069 & 0,083 & 0,087 & 0,085 & 0,090 & 0,100 & $0,086^{\mathrm{e}}$ \\
M Ødias & $0,084^{\mathrm{D}}$ & $0,095^{\mathrm{C}}$ & $0,105^{\mathrm{AB}}$ & $0,098^{\mathrm{BC}}$ & $0,105^{\mathrm{ABC}}$ & $0,117^{\mathrm{A}}$ & \\
\hline F(Trat.) \\
F( poca)
\end{tabular}

* significativo ao nível de $1 \%$.

Médias seguidas de mesma letra não diferem entre si, ao nível de 5\%.

TABELA 7b - ACIDEZ TOTAL TITULÁVEL (g de ácido oxálico/100 g de amostra) DAS CARAMBOLAS ÁCIDAS EM CALDA

\begin{tabular}{c|c|c|c|c|c|c|c}
\hline poca & T1 & T2 & T3 & T4 & T5 & T6 & M Ødias \\
\hline $\mathbf{3 0}$ & 0,182 & 0,184 & 0,211 & 0,204 & 0,216 & 0,231 & $0,205^{\mathrm{ab}}$ \\
$\mathbf{6 0}$ & 0,186 & 0,189 & 0,193 & 0,198 & 0,211 & 0,243 & $0,203^{\mathrm{ab}}$ \\
$\mathbf{9 0}$ & 0,173 & 0,182 & 0,186 & 0,202 & 0,213 & 0,247 & $0,200^{\mathrm{ab}}$ \\
$\mathbf{1 2 0}$ & 0,175 & 0,189 & 0,184 & 0,193 & 0,211 & 0,216 & $0,195^{\mathrm{ab}}$ \\
$\mathbf{1 5 0}$ & 0,182 & 0,193 & 0,191 & 0,195 & 0,198 & 0,227 & $0,198^{\mathrm{b}}$ \\
$\mathbf{1 8 0}$ & 0,164 & 0,164 & 0,180 & 0,175 & 0,195 & 0,245 & $0,187^{\mathrm{b}}$ \\
MØdias & $0,177^{\mathrm{D}}$ & $0,183^{\mathrm{D}}$ & $0,191^{\mathrm{CD}}$ & $0,194^{\mathrm{BC}}$ & $0,207^{\mathrm{AB}}$ & $0,235^{\mathrm{A}}$ & \\
\hline F(Trat.) & \multicolumn{7}{|c}{$24,81^{*}$} \\
F( poca) & $4,25^{*}$ \\
\hline
\end{tabular}

* significativo ao nível de $1 \%$.

Médias seguidas pela mesma letra não diferem entre si ao nível de 5\%.

\subsubsection{Análise Sensorial}

Quanto à textura (Tabelas $8 \mathrm{a}$ e $8 \mathrm{~b}$ ) das frutas doces maiores resultados foram observados aos 30, 60 e 90 dias de armazenamento e para o tipo 
ácido aos 0, 30 e 60 dias. Para as frutas doces não houve diferença significativa entre os tratamentos, embora o 4 (fruta + xarope 22 Brix + $0,1 \%$ de $\mathrm{CaCl}_{2}$ ) tenha revelado maior nota. Já, para o tipo ácido, a maior nota foi recebida pelo tratamento 2 (fruta + xarope 25 Brix $+0,1 \%$ de pectina) que não diferiu do 3 , mas dos demais. Nesse caso, o tratamento 6 recebeu menor nota, embora não tenha diferido do 1, do 4 e do 5.

\section{TABELA 8a-AVALIAÇÃO SENSORIAL DA TEXTURA DAS CARAMBOLAS DOCES EM CALDA}

\begin{tabular}{c|c|c|c|c|c|c|c}
\hline poca & T1 & T2 & T3 & T4 & T5 & T6 & M Ød ia s \\
\hline $\mathbf{0}$ & 6,06 & 5,69 & 6,06 & 4,94 & 4,87 & 5,19 & $5,47^{\mathrm{c}}$ \\
$\mathbf{3 0}$ & 6,81 & 6,81 & 6,56 & 6,62 & 4,75 & 6,00 & $6,26^{\mathrm{ab}}$ \\
$\mathbf{6 0}$ & 6,62 & 6,81 & 6,00 & 6,69 & 7,62 & 5,12 & $6,48^{\mathrm{a}}$ \\
$\mathbf{9 0}$ & 5,37 & 6,62 & 5,94 & 7,25 & 6,75 & 5,94 & $6,31^{\mathrm{ab}}$ \\
$\mathbf{1 2 0}$ & 4,00 & 5,87 & 5,69 & 5,75 & 6,69 & 6,12 & $5,69^{\mathrm{bc}}$ \\
$\mathbf{1 5 0}$ & 5,31 & 5,50 & 5,87 & 6,69 & 5,31 & 6,25 & $5,82^{\mathrm{abc}}$ \\
$\mathbf{1 8 0}$ & 6,12 & 6,12 & 5,06 & 6,25 & 5,94 & 6,12 & $5,93^{\mathrm{abc}}$ \\
M Ødias & $5,75^{\mathrm{A}}$ & $6,20^{\mathrm{A}}$ & $5,88^{\mathrm{A}}$ & $6,31^{\mathrm{A}}$ & $5,99^{\mathrm{A}}$ & $5,82^{\mathrm{A}}$ & \\
\hline F(Trat.) & \multicolumn{7}{|c}{$2,22 \mathrm{n.S}}$. \\
F( poca) & $4,88^{*}$ \\
\hline
\end{tabular}

* significativo ao nível de $1 \%$. n.s. = não significativo ao nível de $5 \%$.

Médias seguidas pela mesma letra não diferem entre si ao nível de 5\%.

TABELA 8b-AVALIAÇÃO SENSORIAL DA TEXTURA DAS CARAMBOLAS ÁCIDAS EM CALDA

\begin{tabular}{c|c|c|c|c|c|c|c}
\hline poca & T1 & T2 & T3 & T4 & T5 & T6 & M Ød ia s \\
\hline $\mathbf{0}$ & 5,37 & 5,31 & 5,25 & 5,00 & 5,25 & 4,94 & $5,19^{\mathrm{ab}}$ \\
$\mathbf{3 0}$ & 5,31 & 5,94 & 5,12 & 4,81 & 4,62 & 5,37 & $5,19^{\mathrm{ab}}$ \\
$\mathbf{6 0}$ & 5,50 & 6,00 & 6,31 & 5,56 & 4,94 & 4,75 & $5,51^{\mathrm{a}}$ \\
$\mathbf{9 0}$ & 5,50 & 5,31 & 4,87 & 4,50 & 4,19 & 3,94 & $4,72^{\mathrm{bc}}$ \\
$\mathbf{1 2 0}$ & 4,69 & 5,50 & 5,62 & 4,19 & 4,94 & 4,44 & $4,90^{\mathrm{abc}}$ \\
$\mathbf{1 5 0}$ & 3,56 & 4,94 & 4,25 & 4,75 & 4,56 & 3,75 & $4,30^{\mathrm{C}}$ \\
$\mathbf{1 8 0}$ & 4,87 & 6,12 & 4,69 & 4,31 & 3,87 & 4,56 & $4,74^{\mathrm{bc}}$ \\
M Ødias & $4,97^{\mathrm{BC}}$ & $5,59^{\mathrm{A}}$ & $5,16^{\mathrm{AB}}$ & $4,73^{\mathrm{BC}}$ & $4,62^{\mathrm{BC}}$ & $4,53^{\mathrm{C}}$ & \\
\hline F(Trat.) & \multicolumn{5}{|l}{$9,20^{*}$} \\
F( poca) & $8,16^{*}$ \\
\hline
\end{tabular}

* significativo ao nível de $1 \%$. 
Médias seguidas pela mesma letra não diferem entre si ao nível de 5\%.

Quanto ao sabor (Tabelas 9a e 9b) os melhores resultados para o tipo doce foram obtidos a partir dos 90 dias de armazenamento. Para o tipo ácido aos 30 dias, diferindo estatisticamente dos demais períodos de armazenamento. Para o tipo doce, o tratamento 6 (fruta + xarope 28 Brix $+0,2 \%$ de pectina $+0,4 \%$ de $\mathrm{CaCl}_{2}+300 \mathrm{mg}$ de ácido ascórbico $/ \mathrm{kg}$ de fruta) destacou-se, diferindo significativamente dos demais. As menores notas foram recebidas pelos tratamentos 1 e 4, que não diferiram entre si, mas dos demais. Para o tipo ácido, a maior nota foi recebida pelo tratamento 3 (fruta + xarope $28 \stackrel{\circ}{\circ}$ Brix $+0,2 \%$ de pectina $+300 \mathrm{mg}$ de ácido ascórbico $/ \mathrm{kg}$ de fruta) que não diferiu de 2 e do 6 , e sim dos demais. Valores inferiores também foram obtidos pelos tratamentos 1 e 4 , que não diferiram entre si e nem do tratamento 5.

\section{TABELA 9a-AVALIAÇÃO SENSORIAL DO SABOR DAS CARAMBOLAS DOCES EM CALDA}

\begin{tabular}{c|c|c|c|c|c|c|c}
\hline poca & T1 & T2 & T3 & T4 & T5 & T6 & Mødias \\
\hline $\mathbf{0}$ & 5,50 & 5,81 & 4,94 & 5,06 & 4,75 & 5,19 & $5,21^{\mathrm{b}}$ \\
$\mathbf{3 0}$ & 5,56 & 6,00 & 5,69 & 4,94 & 5,50 & 5,50 & $5,53^{\mathrm{b}}$ \\
$\mathbf{6 0}$ & 4,44 & 5,19 & 5,81 & 4,69 & 5,69 & 6,19 & $5,33^{\mathrm{b}}$ \\
$\mathbf{9 0}$ & 6,19 & 6,25 & 6,50 & 5,94 & 7,31 & 7,69 & $6,65^{\mathrm{a}}$ \\
$\mathbf{1 2 0}$ & 5,00 & 6,06 & 5,87 & 4,06 & 5,87 & 7,19 & $5,67^{\mathrm{b}}$ \\
$\mathbf{1 5 0}$ & 5,12 & 6,62 & 7,56 & 4,94 & 7,25 & 7,94 & $6,57^{\mathrm{a}}$ \\
$\mathbf{1 8 0}$ & 5,69 & 6,94 & 7,06 & 4,69 & 6,75 & 7,44 & $6,43^{\mathrm{a}}$ \\
Mødias & $5,33^{\mathrm{C}}$ & $6,18^{\mathrm{B}}$ & $6,41^{\mathrm{B}}$ & $4,88^{\mathrm{C}}$ & $6,39^{\mathrm{B}}$ & $6,82^{\mathrm{A}}$ & \\
\hline F(Trat.) & \multicolumn{7}{|c}{$26,96^{*}$} \\
F( poca) & $20,08^{*}$
\end{tabular}

* significativo ao nível de $1 \%$.

Médias seguidas pela mesma letra não diferem entre si ao nível de 5\%.

Com relação à cor (Tabelas 10a e 10b), para as frutas do tipo doce não ocorreram diferenças significativas entre as épocas de avaliação do produto. Para o tipo ácido a maior nota foi observada aos 150 dias de armazenamento. A pior nota foi observada no tempo zero, que diferiu significativamente das demais. O tratamento 1 (fruta + xarope $22{ }^{\circ}$ Brix) para as frutas doces apresentou maior resultado, diferindo dos demais. $A$ menor nota foi recebida pelo tratamento 4 , que também diferiu dos outros. Já para o tipo ácido, as maiores notas foram obtidas pelos tratamentos 4 
(fruta + xarope $22{ }^{\circ} \mathrm{Brix}+0,1 \%$ de $\mathrm{CaCl}_{2}$ ), 5 (fruta + xarope $25{ }^{\circ} \mathrm{Brix}+$ $0,1 \%$ de pectina $+0,2 \%$ de $\mathrm{CaCl}_{2}$ ) e 6 (fruta + xarope $28{ }^{\circ} \mathrm{Brix}+0,2 \%$ de pectina $+0,4 \%$ de $\mathrm{CaCl}_{2}+300 \mathrm{mg}$ de ácido ascórbico $/ \mathrm{kg}$ de fruta), que não diferiram entre si, mas dos demais. Nesse caso, o tratamento 1 apresentou menor nota, e diferiu dos outros.

\section{TABELA 9b-AVALIAÇÃO SENSORIAL DO SABOR DAS CARAMBOLAS ÁCIDAS EM CALDA}

\begin{tabular}{c|c|c|c|c|c|c|c}
\hline poca & T1 & T2 & T3 & T4 & T5 & T6 & M Ødias \\
\hline $\mathbf{0}$ & 5,37 & 5,69 & 5,50 & 5,31 & 6,06 & 6,12 & $5,67^{\mathrm{b}}$ \\
$\mathbf{3 0}$ & 6,50 & 6,44 & 6,62 & 6,75 & 6,31 & 6,94 & $6,59^{\mathrm{a}}$ \\
$\mathbf{6 0}$ & 5,62 & 5,87 & 5,12 & 5,44 & 5,81 & 6,06 & $5,82^{\mathrm{b}}$ \\
$\mathbf{9 0}$ & 5,25 & 5,44 & 5,87 & 5,06 & 5,25 & 4,94 & $5,30^{\mathrm{b}}$ \\
$\mathbf{1 2 0}$ & 4,44 & 5,69 & 6,56 & 4,50 & 5,37 & 5,62 & $5,36^{\mathrm{b}}$ \\
$\mathbf{1 5 0}$ & 4,06 & 5,75 & 6,50 & 4,69 & 5,44 & 6,00 & $5,41^{\mathrm{b}}$ \\
$\mathbf{1 8 0}$ & 4,50 & 5,62 & 6,81 & 4,69 & 5,56 & 5,62 & $5,47^{\mathrm{b}}$ \\
MØdias & $5,10^{\mathrm{C}}$ & $5,78^{\mathrm{AB}}$ & $6,14^{\mathrm{A}}$ & $5,20^{\mathrm{C}}$ & $5,68^{\mathrm{BC}}$ & $5,90^{\mathrm{AB}}$ & \\
\hline F(Trat.) & \multicolumn{7}{|l}{$9,18^{*}$} \\
F( poca) & \multicolumn{7}{|l}{}
\end{tabular}

${ }^{*}$ significativo ao nível de $1 \%$

Médias seguidas pela mesma letra não diferem entre si, ao nível de 5\%.

TABELA 10a - AVALIAÇÃO SENSORIAL DA COR DAS CARAMBOLAS DOCES EM CALDA

\begin{tabular}{c|c|c|c|c|c|c|c}
\hline poca & T1 & T2 & T3 & T4 & T5 & T6 & M Ødias \\
\hline $\mathbf{0}$ & 6,87 & 5,87 & 5,75 & 4,50 & 4,00 & 3,87 & $5,14^{\mathrm{a}}$ \\
$\mathbf{3 0}$ & 6,87 & 5,25 & 5,37 & 2,69 & 5,44 & 4,19 & $4,97^{\mathrm{a}}$ \\
$\mathbf{6 0}$ & 5,75 & 4,00 & 5,81 & 2,56 & 3,25 & 7,31 & $4,78^{\mathrm{a}}$ \\
$\mathbf{9 0}$ & 7,12 & 4,81 & 5,87 & 1,87 & 4,06 & 7,37 & $5,18^{\mathrm{a}}$ \\
$\mathbf{1 2 0}$ & 6,94 & 3,94 & 7,31 & 1,62 & 4,75 & 4,62 & $4,86^{\mathrm{a}}$ \\
$\mathbf{1 5 0}$ & 5,94 & 3,19 & 4,44 & 1,62 & 6,25 & 5,31 & $4,46^{\mathrm{a}}$ \\
$\mathbf{1 8 0}$ & 6,50 & 5,94 & 6,31 & 2,06 & 4,44 & 5,50 & $5,12^{\mathrm{a}}$ \\
Mødias & $6,52^{\mathrm{A}}$ & $4,52^{\mathrm{C}}$ & $5,85^{\mathrm{B}}$ & $2,07^{\mathrm{D}}$ & $4,70^{\mathrm{C}}$ & $5,72^{\mathrm{B}}$ & \\
\hline F(Trat.) & \multicolumn{7}{|c}{2,27 n.s. } \\
F( poca) & \multicolumn{7}{|l}{}
\end{tabular}

* significativo ao nível de $1 \%$. n.s. - não significativo ao nível de $5 \%$. Médias seguidas pela mesma letra não diferem entre si ao nível de 5\%. 
TABELA 10b - AVALIAÇÃO SENSORIAL DA COR DAS CARAMBOLAS ÁCIDAS EM CALDA

\begin{tabular}{c|c|c|c|c|c|c|c}
\hline poca & T1 & T2 & T3 & T4 & T5 & T6 & M Ødia s \\
\hline $\mathbf{0}$ & 6,19 & 6,00 & 5,62 & 4,06 & 4,37 & 2,94 & $4,86^{\mathrm{c}}$ \\
$\mathbf{3 0}$ & 4,44 & 5,94 & 5,87 & 6,69 & 6,12 & 7,25 & $6,05^{\mathrm{ab}}$ \\
$\mathbf{6 0}$ & 5,06 & 5,00 & 5,25 & 7,12 & 7,19 & 7,44 & $6,18^{\mathrm{ab}}$ \\
$\mathbf{9 0}$ & 4,06 & 5,50 & 6,12 & 6,37 & 7,31 & 6,56 & $5,99^{\mathrm{ab}}$ \\
$\mathbf{1 2 0}$ & 3,62 & 5,56 & 4,69 & 7,12 & 7,81 & 8,06 & $6,14^{\mathrm{ab}}$ \\
$\mathbf{1 5 0}$ & 4,44 & 6,12 & 5,25 & 8,06 & 7,81 & 8,87 & $6,76^{\mathrm{a}}$ \\
$\mathbf{1 8 0}$ & 5,19 & 5,62 & 5,87 & 5,75 & 6,75 & 6,00 & $5,86^{\mathrm{b}}$ \\
M Ødias & $4,46^{\mathrm{C}}$ & $5,46^{\mathrm{B}}$ & $5,51^{\mathrm{B}}$ & $6,85^{\mathrm{A}}$ & $7,16^{\mathrm{A}}$ & $7,36^{\mathrm{A}}$ & \\
\hline F(Trat.) & \multicolumn{7}{|l}{$26,62^{*}$} \\
F( poca) & $10,87^{*}$ \\
\hline
\end{tabular}

* significativo ao nível de $1 \%$.

Médias seguidas pela mesma letra não diferem entre si, ao nível de 5\%.

Comparando-se as médias dos tratamentos dos dois tipos de fruta, em relação às avaliações sensoriais de textura, sabor e cor do produto processado termicamente, o tipo doce apresentou para textura e sabor maiores notas. No entanto, quanto ao atributo cor foi o tipo ácido que alcançou notas superiores. De acordo com a Tabela 11 pode-se afirmar que, em relação ao tipo doce não seriam recomendados os tratamentos 1 para o parâmetro textura e 4 para os parâmetros sabor e cor. Em relação ao tipo ácido não seriam recomendados os tratamentos 6 para textura e 1 para sabor e cor.

TABELA 11 - AVALIAÇÃO SENSORIAL DAS CARAMBOLAS EM CALDA

\begin{tabular}{c|c|c|c|c|c|c}
\hline \multicolumn{9}{c}{ Mødia das Avalia I es } \\
\hline & \multicolumn{2}{|c|}{ Textura } & \multicolumn{2}{c}{ Sabor } & \multicolumn{2}{c}{ Cor } \\
\hline Tratamentos & Doce & cida & Doce & cida & Doce & cida \\
\hline 1 & $5,75^{\mathrm{A}}$ & $4,97^{\mathrm{BC}}$ & $5,36^{\mathrm{C}}$ & $5,10^{\mathrm{C}}$ & $6,57^{\mathrm{A}}$ & $4,71^{\mathrm{C}}$ \\
2 & $6,20^{\mathrm{A}}$ & $5,59^{\mathrm{A}}$ & $6,12^{\mathrm{B}}$ & $5,78^{\mathrm{AB}}$ & $4,71^{\mathrm{C}}$ & $5,68^{\mathrm{B}}$ \\
3 & $5,88^{\mathrm{A}}$ & $5,16^{\mathrm{AB}}$ & $6,20^{\mathrm{B}}$ & $6,14^{\mathrm{A}}$ & $5,84^{\mathrm{B}}$ & $5,52^{\mathrm{B}}$ \\
4 & $6,31^{\mathrm{A}}$ & $4,73^{\mathrm{BC}}$ & $4,90^{\mathrm{C}}$ & $5,20^{\mathrm{C}}$ & $2,42^{\mathrm{D}}$ & $6,45^{\mathrm{A}}$ \\
5 & $5,99^{\mathrm{A}}$ & $4,62^{\mathrm{BC}}$ & $6,16^{\mathrm{B}}$ & $5,68^{\mathrm{BC}}$ & $4,60^{\mathrm{C}}$ & $6,76^{\mathrm{A}}$ \\
6 & $5,82^{\mathrm{A}}$ & $4,53^{\mathrm{C}}$ & $6,73^{\mathrm{A}}$ & $5,90^{\mathrm{AB}}$ & $5,45^{\mathrm{B}}$ & $6,73^{\mathrm{A}}$ \\
\hline F (trat.) & $2,22^{\mathrm{n} . S}$ & $9,20^{*}$ & $26,96^{*}$ & $9,18^{*}$ & $77,95^{*}$ & $26,62^{*}$ \\
\hline
\end{tabular}

n.s. = não significativo ao nível de $5 \%$.

* significativo ao nível de $1 \%$.

Médias seguidas pela mesma letra não diferem entre si ao nível de 5\%. 


\section{CONCLUSÃO}

Em relação à textura, para o produto obtido com as frutas doces, o melhor tratamento foi o que empregou fruta, xarope a 28 Brix, $0,2 \%$ de pectina, $0,4 \%$ de $\mathrm{CaCl}_{2}$, e $300 \mathrm{mg}$ de ácido ascórbico/kg de fruta. Para o tipo ácido foi o que empregou fruta, xarope a $25^{\circ}$ Brix e $0,1 \%$ de pectina.

A fruta do tipo doce (tanto processada como "in natura") apresentou melhor textura, não sendo efetiva a adição de $\mathrm{CaCl}_{2}$ para a manutenção da firmeza das frutas ácidas.

Os teores de ácido ascórbico diminuíram com o decorrer do tempo de armazenamento. Foi observado que mesmo após 180 dias, os teores de ácido ascórbico mostraram-se bastante satisfatórios (32,91 mg/100 g para o tipo doce, e 36,49 mg/100 g para o tipo ácido).

Segundo as análises sensoriais as frutas do tipo doce destacaram-se pela textura e sabor, enquanto que o tipo ácido pela cor.

Os melhores tratamentos em relação às análises sensoriais de textura, sabor e cor para as frutas do tipo doce foram, respectivamente, os que receberam: fruta, xarope a $22{ }^{\circ} \mathrm{Brix}$ e $0,1 \%$ de $\mathrm{CaCl}_{2}$; fruta, xarope a 28 Brix, 0,2\% de pectina, 0,4\% de $\mathrm{CaCl}_{2}$, e $300 \mathrm{mg}$ de ácido ascórbico/ $\mathrm{kg}$ de fruta; fruta e xarope a 22 Brix. Para o tipo ácido, os melhores tratamentos foram, respectivamente, os que receberam: fruta, xarope a $25 \stackrel{\circ}{\circ}$ Brix e $0,1 \%$ de pectina; fruta, xarope a 28 Brix, $0,2 \%$ de pectina, e $300 \mathrm{mg}$ de ácido ascórbico/kg de fruta; fruta, xarope a 25 Brix, 0,1\% de pectina e $0,2 \%$ de $\mathrm{CaCl}_{2}$.

Os resultados obtidos neste trabalho sugerem que as carambolas do tipo doce são as mais indicadas para elaboração de fruta em calda. Também permitem recomendar a realização de estudos mais detalhados sobre o processamento de carambola em calda, bem como pesquisas sobre outros tipos de conserva para melhor aproveitamento da fruta.

\section{Abstract}

EVALUATION OF STAR FRUIT (AVERRHOA CARAMBOLA L.) SWEET AND SOUR FOR THE CANNED PROCESSING

Two kinds of star fruit (sweet and acid) were analyzed physically (texture and yield), and chemically ( $\mathrm{pH}$, titratable acidity, ascorbic acid and soluble solids content), in order to determine their suitability for canned fruit processing. Different chemical treatments 
were used for processing the canned fruit (use of syrups with different saccharose concentrations, addition of pectin and $\mathrm{CaCl}_{2}$ in different concentrations and ascorbic acid), in order to evaluate the adequability of the two kinds of star fruit for thermal processing. The products were submitted to physical analysis (gross weight, drained weight, vacuum and texture), chemical analysis ( $\mathrm{pH}$, titratable acidity, ascorbic acid and soluble solids content), and evaluated sensorially (texture, flavor and color). Regarding the product obtained with the sweet fruits, the addition of $\mathrm{CaCl}_{2}$ and pectin was effective in the maintenance of its texture; for the product obtained with the acid kind this practice didn't produce the same effect, considering that the treatments added with $\mathrm{CaCl}_{2}$ were evaluated as inferior to that whose syrup was added with pectin, what indicates that this component is the responsable for the good texture in the processed acid fruits. Both kinds of star fruit studied presented, under a technologic point of view, good physical and chemical characteristics, but the sweet kind of fruits were more apropriated for processing canned fruit, being superior in terms of texture and flavor.

KEY WORDS: STAR FRUIT-PROCESSING; CANNED FRUIT.

\section{REFERÊNCIAS}

1 AOAC. Association of Official Analitycal Chemists. Official methods of analysis of AOAC Internacional. $16^{\text {th }}$ ed. Gaithsburg, 1997. v. 2, Cap. 37 (Fruits and fruit products).

2 AWAD, M. Fisiologia pós-colheita de frutos. São Paulo: Nobel, 1993. $114 \mathrm{p}$.

3 BAUMGARTNER, J.G.; HERSON, A.C. Conservas alimentícias. Zaragoza: Acribia, 1959. p. 149-180. Cap.9 (Princípios del proceso de enlatado de los alimentos).

4 CAMPBELL, C.A.; KOCH, K.E. Acid composition and development of sweet and tart carambola fruit. Journal of American Society of Horticultural Science, v.114, n.3, p.455-457, 1989.

5 ELEUTÉRIO, R.M.C. Variação do teor de ácido ascórbico e de b - caroteno em suco e geléia de acerola (Malpiguia glabra L. - sinônimo M. punicifolia L.) durante o armazenamento. Piracicaba, 1998. 77 p. Dissertação (Mestrado) - Escola Superior de Agricultura “Luiz de Queiroz", Universidade de São Paulo.

6 ESKIN, N.A.M.; HENDERSON, H.M.; TOWNSEND, R.J. Biochemistry of foods. New York: Academic Press, 1971. 240p.

7 FONSECA, H. Conservação pelo calor. In: FILHO, A.M.; OLIVEIRA, 
A.J. de; FONSECA, H. et al. Processamento e conservação de alimentos. Piracicaba: ESALQ/Departamento de Ciência e Tecnologia de Alimentos, 1976. Cap. 2.

8 FONSECA, H. Princípios e métodos gerais de conservação de alimentos - conservação pelo calor e pelo frio. In: CAMARGO, R. Tecnologia dos produtos agropecuários - alimentos. São Paulo: Nobel, 1984. p.73-95.

9 FONSECA, H.; NOGUEIRA, J.N.; Processamento e conservação de alimentos de origem vegetal - frutas. In: CAMARGO, R. Tecnologia dos produtos agropecuários - alimentos. São Paulo: Nobel, 1984. p.113-124.

10 HOLDSWORTH, S.D. Conservacion de frutas y hortalizas. Zaragoza: Acribia, 1988. $186 \mathrm{p}$.

11 IADEROZA, M.; DRAETTA, I.S. Ênzimos e pigmentos - influências e alterações durante o processamento. In: SOLER, M.P.; BLEINROTH, E.W.; IADEROZA, M.; DRAETTA, I.S. Industrialização de frutas. 2.ed. Campinas: ITAL/Rede de informação de tecnologia industrial básica, 1991. p.17-31. Cap.4 (Manual Técnico, 8).

12 JOSEPH, J.; MENDONCA, G. Chemical characteristics of Averrhoa carambola L. Proceedings of Interamerican Society of Tropical Horticulture, n.33, p.111-116, 1989a.

13 JOSEPH, J.; MENDONCA, G. Studies on the candying process of Averrhoa carambola $\mathrm{L}$. and the utilization of the product in bakery products and desserts. Proceedings of Interamerican Society of Tropical Horticulture, n.33, p.121-126, 1989b.

14 KERTESZ, Z.I. The pectic substances. New York: Interscience Publishers, $1951.628 \mathrm{p}$.

15 KISS, E. Carambola: pomar de estrelas. Globo Rural, n.150, p.3134, abr. 1998.

16 LEME JÚNIOR, J.; MALAVOLTA, E. Determinação fotométrica de ácido ascórbico. Anais da Escola Superior de Agricultura "Luiz de Queiroz", v.7., p.115-129, 1950. 
17 MARTIN, S.L. Selection and training of sensory judges. Food Technology, v.27, n.11, p.22-26, 1973.

18 MATTHEWS, R.F. Processing of carambola. Proceedings of Interamerican Society of Tropical Horticulture, n.33, p.83-90, 1989.

19 MONTGOMERY, M.W. Ênzimos importantes no processamento de alimentos. In: IADEROZA, M.; BALDINI, V.L.S. Ênzimos e a qualidade de vegetais processados. Campinas: ITAL, 1991. p.2336. (Manual Técnico)

20 MORI, E.E.M. Reologia e textura do ponto de vista organoléptico. In: CAMPOS, S.D.S.; GONÇALVES, J.R.; MORI, E.E.M.; GASPARETTO, C.A.; Reologia e textura de alimentos. Campinas: ITAL, 1989. p.17-42.

21 NAGY, S.; BARROS, S.; CARTER, R. Production and characterization of carambola essence. Proceedings Florida State Horticultural Science, n.103, p.277-279, 1990.

22 NOVAES, L.C.C. Avaliação da qualidade dos néctares comum e dietético de morango das variedades Guarani e Oso Grande. Piracicaba, 1997. 119p. Dissertação (Mestrado) - Escola Superior de Agricultura “Luiz de Queiroz", Universidade de São Paulo.

23 OLIVEIRA, M.N. de; MAIA, G.A.; GUEDES, Z.B. de L.; GUIMARÃES, A.C.L.; FIGUEIREDO, R.W. de Características químicas e físicoquímicas da carambola (Averrhoa carambola L.). Ciências Agronômicas, v.20, n.1/2, p.129-133, jul./dez. 1989.

24 PASCHOALINO, J.E. Controle das reações catalisadas pelos ênzimos. In: IADEROZA, M.; BALDINI, V.L.S. Ênzimos e a qualidade de vegetais processados. Campinas: ITAL, 1991. p.5358. (Manual Técnico).

25 PRADO FILHO, L.G. do; PRADO, M.C. Carotenóides: estrutura química, obtenção e emprego em alimentos. Piracicaba: ESALQ/ Departamento de Ciência e Tecnologia de Alimentos, 1992. 42 p.

26 RICHARDS, N.S.P.S. Avaliação da qualidade das variedades Chandler e Reiko para a obtenção de morango em calda. 
Piracicaba, 1996. 90 p. Tese (Dissertação) - Escola Superior de Agricultura "Luiz de Queiroz", Universidade de São Paulo.

27 SALOMÓN, E.A.G.; MARTIN, Z.J.; KATO, K. et al. Formulações de néctares de frutas tropicais. Boletim do Instituto de Tecnologia de Alimentos, n.50, p.103-121, mar./abr. 1977.

28 SAS Institute. SAS User's Guide: statistics. Cary, USA, 1993.

29 SIVIERI, K. Avaliação da qualidade de geléias de morango comum e dietética das variedades Guarani e Oso Grande. Piracicaba, 1997. 124 p. Dissertação (Mestrado) - Escola Superior de Agricultura "Luiz de Queiroz", Universidade de São Paulo.

30 SOLER, M.P.; BLEINROTH, E.W.; IADEROZA, M. et al. Industrialização de frutas. 2.ed. Campinas: ITAL/Rede de informação de tecnologia industrial básica, 1991. 206 p. (Manual Técnico, 8). 